\title{
Comparative Efficacy and Safety of Long-acting Injectable and Oral Second-generation Antipsychotics for the Treatment of Schizophrenia: A Systematic Review and Meta-analysis
}

\author{
Seon-Cheol Park ${ }^{1, *}$, Mi Young $\mathrm{Choi}^{2}, *$, Jina Choi ${ }^{2}$, Eunjung Park' ${ }^{2}$, Ha Jin Tchoe $^{2}$, Jae Kyung Suh ${ }^{2}$, \\ Young Hoon Kim ${ }^{3}$, Seung Hee Won ${ }^{4}$, Young-Chul Chung ${ }^{5}$, Kyung-Yeol Bae ${ }^{6}$, Sang-Kyu Lee, Chan Mi Park ${ }^{2}$, \\ Seung-Hwan Lee $^{8}$ \\ ${ }^{1}$ Department of Psychiatry, Haeundae Paik Hospital, Inje University College of Medicine Busan, ${ }^{2}$ National Evidence-based Healthcare \\ Collaborating Agency, Seoul, ${ }^{3}$ Department of Psychiatry, Gongju National Hospital, Gongju, ${ }^{4}$ Department of Psychiatry, School of Medicine, \\ Kyungpook National University, Daegu, ${ }^{5}$ Department of Psychiatry, Chonbuk National University Hospital, Jeonju, ${ }^{6}$ Department of Psychiatry, \\ Chonnam National University Medical School, Gwangju, ${ }^{7}$ Department of Psychiatry, Hallym University Chuncheon Sacred Hospital, Hallym \\ University College of Medicine, Chuncheon, ${ }^{8}$ Department of Psychiatry, Inje University Ilsan Paik Hospital, Goyang, Korea
}

\begin{abstract}
We aimed to compare the efficacy and safety of long-acting injectable (LAI) and oral second-generation antipsychotics (SGAs) in treating schizophrenia by performing a systematic review and meta-analysis. MEDLINE, EMBASE, PsycINFO, $\mathrm{CINAHL}$, and the Cochrane Library, as well as five Korean databases, were systemically searched to identify studies published from 2000 to 16 April 2015, which compared the efficacy and safety of LAI and oral SGAs. Using data from randomized controlled trials (RCTs), meta-analyses were conducted. In addition, the GRADE (the Grading of Recommendations, Assessment, Development and Evaluation) approach was applied to explicitly assess the quality of the evidence. A total of 30 studies including 17 RCTs and 13 observational studies were selected. The group treated with LAI SGAs was characterized by significantly lower relapse rates, longer times to relapse and fewer hospital days, but also by a higher occurrence of extrapyramidal syndrome and prolactin-related symptoms than that in the group treated with oral SGAs. Our findings demonstrate that there is moderate to high level of evidence suggesting that in the treatment of schizophrenia, LAI SGAs have higher efficacy and are associated with higher rates of extrapyramidal syndrome and prolactin-related symptoms. Additionally, the use of LAI SGAs should be combined with appropriate measures to reduce dopamine $\mathrm{D}_{2}$ antagonism-related symptoms.
\end{abstract}

KEY WORDS: Efficacy; Long-acting injectable; Safety; Schizophrenia; Second-generation antipsychotics.

\section{INTRODUCTION}

In terms of the global burden of mental disorders, schizophrenia has been identified as an important mental

Received: February 1, 2018/ Accepted: March 5, 2018 Address for correspondence: Seung-Hwan Lee, MD, PhD Department of Psychiatry, Ilsan Paik Hospital, Inje University College of Medicine, 170 Juhwa-ro, Ilsanseo-gu, Goyang 10380, Korea

Tel: +82-31-910-7260, Fax: +82-31-910-7268

E-mail: Ishpss@hanmail.net

ORCID: https://orcid.org/0000-0003-0305-3709

Chan Mi Park, PhD

National Evidence-based Healthcare Collaborating Agency,

Namasan Square (Kukdong B/D) 7F, 173 Toegye-ro, Jung-gu,

Seoul 04554, Korea

Tel: +82-2-2174-2720, Fax: +82-2-747-4916

E-mail: chpark@neca.re.kr

*These authors contributed equally to this study as co-first authors. health issue by the Grand Challenges in Global Mental Health Initiative. ${ }^{1-4)}$ Antipsychotic medication non-compliance occurs in $80 \%$ or more of patients with schizophrenia, and can last for substantial periods of time, despite recommendations from clinical psychiatrists. ${ }^{5)}$ The European Schizophrenia Health Outcome Study (SOHO) demonstrated that non-compliance was associated with recurrence risk, hospitalization rate, and suicidal risk, among more than 7,000 patients with schizophrenia. ${ }^{6)}$ In addition, antipsychotic non-compliance has been shown to be significantly associated with an increased risk of rehospitalization, emergency room visits, homelessness, and symptom exacerbation." Hence, "long-acting injectable" (LAI) or depot antipsychotics have been proposed as one of the most promising potential alternative

(c) This is an Open-Access article distributed under the terms of the Creative Commons Attribution Non-Commercial License (http://creativecommons.org/licenses/by-nc/4.0) which permits unrestricted non-commercial use, distribution, and reproduction in any medium, provided the original work is properly cited. 
treatments to overcome non-compliance in patients with schizophrenia. A meta-analysis of 10 randomized clinical trials lasting at least 12 months, and including 1,700 patents with schizophrenia showed that LAI antipsychotics are associated with significant reductions in relapses and dropouts due to the lack of efficacy when compared with oral antipsychotics. ${ }^{8)}$

The efficacy and tolerability profiles of LAI and oral antipsychotics have been compared in several studies using a systematic review and/or meta-analysis approach. ${ }^{9-17)}$ Most of the systematic reviews and meta-analyses conceptualized LAI and oral antipsychotics as the intervention and the comparator, respectively, without regard for whether the medications were classified as first-generation antipsychotics (FGAs) or second-generation antipsychotics (SGAs). In terms of FGAs and SGAs, "atypicality", which is associated with SGAs, can be defined as a favorable antipsychotic effect with a reduced risk of extrapyramidal side effects. ${ }^{18-21)}$ Moreover, LAI FGAs are rarely used in real-world psychiatry and, particularly in the United States, only haloperidol decanoate and fluphenazine decanoate are used. ${ }^{22)}$ However, since most of the systematic reviews and meta-analyses concerning the efficacy and safety of LAI antipsychotics have been evaluated without regard for the distinction of LAI FGAs and SGAs, to our knowledge, the comprehensive efficacy and safety of LAI SGAs in real-world settings have not been well reported. In addition, extrapyramidal side effects and other adverse effects may be associated with discontinuation of antipsychotics in patients with schizophrenia. ${ }^{15,23)}$ The present systematic review and metaanalysis aimed to compare overall profiles for both efficacy (relapse rate, time to relapse, hospitalization rate, remission rate, hospital days, non-compliance, quality of life, and changes in symptom severity) and safety (all cause discontinuation, extrapyramidal side effects, abnormal involuntary movements, and other variables) of LAI and oral SGAs among schizophrenia patients.

\section{METHODS}

\section{Study Overview}

A systematic search was performed using international databases including MEDLINE, EMBASE, the Cochrane Library, CINAHL, and PsycINFO, and also several Korean domestic databases, including KoreaMed, Korean Medicine
Database (KMBASE), Korean Studies Information Service System (KISS), National Digital Science Library (NDSL), and the Research Information Sharing Service (RISS), with a date range limit for publications from 2000 to 16 April 2015. The key questions and the PICO framework (Patient, Intervention, Comparator, Outcome) ${ }^{24)}$ were defined with the advice of an expert group of psychiatry specialists in the field of schizophrenia research $(\mathrm{KYH}, \mathrm{WSH}$, CYC, BKY, LSK, PSC, and LSH). Extensive searches of the databases using the terms "schizophrenia," "Iong acting injection," and each of the generic names of the SGAs, as defined by Tandon et al., ${ }^{25)}$ were performed. Terms were related to the databases' index terms, such as Medical Subject Headings (MeSH) and EMTREE.

\section{Eligibility Criteria}

Articles that met the following criteria were included: 1) study subjects were adults with schizophrenia (including initial and relapsed patients); 2) the intervention and the active comparator were LAI and oral SGAs, respectively; 3) at least one of the predetermined outcomes was reported; 4) the study was designed as a randomized controlled trial (RCT), non-RCT, cohort study, case-control study, or mirror-image study; and 5) articles were published in English or Korean. Articles that met the following criteria were excluded: 1 ) reports on animal trials or preclinical studies, and non-original research articles such as reviews, editorials, letters, and comments; 2) articles not published in English or Korean, and those with duplicate subjects (studies using the same outcome indicators and publishing in duplicate) were also excluded.

\section{Outcome Measures}

Relapse rate, non-compliance rate, time to relapse, hospitalization rate, remission rate, hospital days, quality of life, and changes in assessment scales, including the Clinical Global Impression-Severity (CGI-S), ${ }^{26)}$ Positive and Negative Syndrome Scale (PANSS), ${ }^{27)}$ and the Montgomery-Asberg Depression Rating Scale (MADRS) ${ }^{28)}$ were used as measures of efficacy. The discontinuation rate, drug-related side effects, and changes in assessment scales, including the Extrapyramidal Symptom Rating Scale (ESRS), ${ }^{29)}$ and the Abnormal Involuntary Movement Scale (AIMS) ${ }^{30)}$ were used as safety measures. 


\section{Selection of Studies and Quality Assessment}

Study selection was initially conducted by the researchers in pairs (CMY, PSC, THJ, CJ, PCM, SJK, PE, and LSH). Namely, screening of all studies by title and abstract, and selection of studies according to the eligibility criteria were conducted by independent pairs of investigators. Selected studies were then discussed and agreed upon in consensus meetings, with consultation from the expert group. The literature selection process was conducted in accordance with the "preferred reporting items for systematic reviews and meta-analysis protocols" (PRISMA-P) 2015 statement. ${ }^{31)}$ Quality assessment of the selected studies was conducted using the Cochrane Risk of Bias Tools ${ }^{32)}$ for the RCTs, and the revised risk of bias tool for non-randomized studies (RoBANS), ${ }^{33)}$ for the observational studies. Quality assessments were conducted by independent pairs of investigators and a consensus was achieved by discussion, with consultation from the expert group.

\section{Data Extraction and Management}

According to the pre-defined data extraction format, researchers worked in pairs to extract data from the selected studies. Detailed data, including patient characteristics and primary and secondary outcomes, were extracted from the selected studies. Continuous variables such as mean change from baseline, median range and standard deviation were converted according to the formula described in the Cochrane Handbook for Systematic Reviews of Interventions. ${ }^{32)}$ The extracted data were synthesized in terms of quantity and quality.

\section{Data Synthesis and Analysis}

The data analyses were conducted in terms of both research type (RCT vs. observational study) and medication type (LAI vs. oral antipsychotic). The standardized mean difference (SMD) or relative risk (RR) and 95\% confidence interval $(\mathrm{Cl})$ from each of the individual RCTs were calculated, and meta-analysis using the fixed effect model was fitted. The fixed effect model assumed that all of the studies had the same treatment effect, and variation in research findings was assumed to be caused by sampling variation or random effects. Along with an evaluation of publication bias, sensitivity analysis and subgroup analysis were also conducted. The publication bias test (funnel plot asymmetry) was only used when at least 10 studies were included in the meta-analysis. Heterogeneity of effects was evaluated using the Higgins $I^{2}$ statistic. All statistical analyses were performed using Cochrane RevMan version 5.3 (The Nordic Cochrane Centre, The Cochrane Collaboration, Copenhagen, Denmark) and R Software ( $R$ version 3.1.2; R Foundation for Statistical Computing, Vienna, Austria), including meta packages.

\section{Level of Evidence}

The level of evidence was evaluated using the methodology developed by the Grading of Recommendations, Assessment, Development and Evaluation (GRADE) working group. ${ }^{33)}$ The GRADE methodology first evaluates the importance of individual outcomes and then determines the level of evidence for each individual outcome as high, moderate, low, or very low. The factors associated with reducing the level of evidence in terms of the risk of bias were as follows: if the presence of performance bias was considered in an association with the open-label designed studies, the level of evidence was reduced by one level. The Summary of Findings table using the GRADE methodology contained a maximum of seven findings, ranked by importance. Measures of clinical efficacy included relapse rate, time to relapse, hospitalization rate, remission rate, hospital days, non-compliance, and quality of life. Measures of safety outcomes included all-cause discontinuation rate, drug-related side effects, ESRS, AIMS, and other side effects. The level of evidence was evaluated using the methodology developed by the GRADE working group. ${ }^{34)}$ Confidence in the estimate of effect was categorized into four levels, including very low, low, moderate and high. Factors associated with lowering the level of evidence were risk of bias, inconsistency, indirectness, imprecision, and publication bias. For the observational studies, factors associated with heightening the level of evidence were a large magnitude of effect, a dose-response gradient, and an effect of plausible residual confounding. Evidence summary of findings tables for each research question were prepared using GRADEpro (McMaster University, Hamilton, ON, Canada; available at: http://ims.cochrane.org/revman/ other-resources/gradepro/download). 


\section{RESULTS}

\section{Study Characteristics}

A total of 3,062 articles were retrieved from the databases. After exclusion of duplicates, 1,933 articles remained. According to the selection criteria, 17 RCTs and 13 observational studies were selected. ${ }^{35-64)}$ All selection steps are presented as a PRISMA flowchart. Finally, the 17 RCTs are selected for our meta-analysis (Fig. 1).

In terms of the geographical locations in which the studies were conducted, most of the studies $(70.6 \%)$ were conducted internationally (eight studies were conducted globally, three studies were conducted in Europe, and one study was conducted in Asia), $17.6 \%$ of studies were conducted in North America (three studies were conducted in the United States), 5.9\% were conducted in Europe (one study was conducted in the United Kingdom), and $5.9 \%$ were conducted in Asia (one study was conducted in Taiwan). In addition, the follow-up periods for $37 \%$ and $36 \%$ of articles were two years and less than one year, respectively. The periods for $17 \%, 7 \%$, and $3 \%$ of the studies were between one and two years, three years, and five years, respectively. All studies were published between 2005 and 2015 (Table 1).

The LAI antipsychotics used as the intervention treatments in the selected articles included risperidone LAI (70.6\%), olanzapine LAI $(17.6 \%)$, and aripiprazole LAI
(11.8\%). Oral antipsychotics used as active comparators included aripiprazole (23.5\%), olanzapine (23.5\%), quetiapine $(17.6 \%)$, and risperidone $(11.8 \%)$, with an unspecified oral SGA used in the remainder of the studies (23.5\%) (Table 1).

\section{Risk of Bias Assessment of Selected Studies}

Risk of bias for the 17 RCTs was assessed using the Cochrane Risk of Bias Tool. ${ }^{30)}$ Random sequence generation, allocation concealment, incomplete outcome, and selective reporting showed approximately low risks of bias. In contrast, blinding of participants and blinding of outcome assessment showed a high or an unclear risk of bias.

Risk of bias for the 13 observational studies was assessed using the revised RoBANS tool. The results for the risk of bias were as follows: the blinding of outcome assessment presented a high or unclear risk of bias in the prospective studies, whereas participant comparability, selection of participants, and confounding variables presented relatively high risks of bias in the retrospective studies. Blinding of outcome assessment and confounding variables presented unclear risks of bias in the mirror-image studies (Fig. 1).

\section{Efficacy Outcomes}

A summary of the efficacy outcomes, including relapse rate, non-compliance, time to relapse, hospitalization

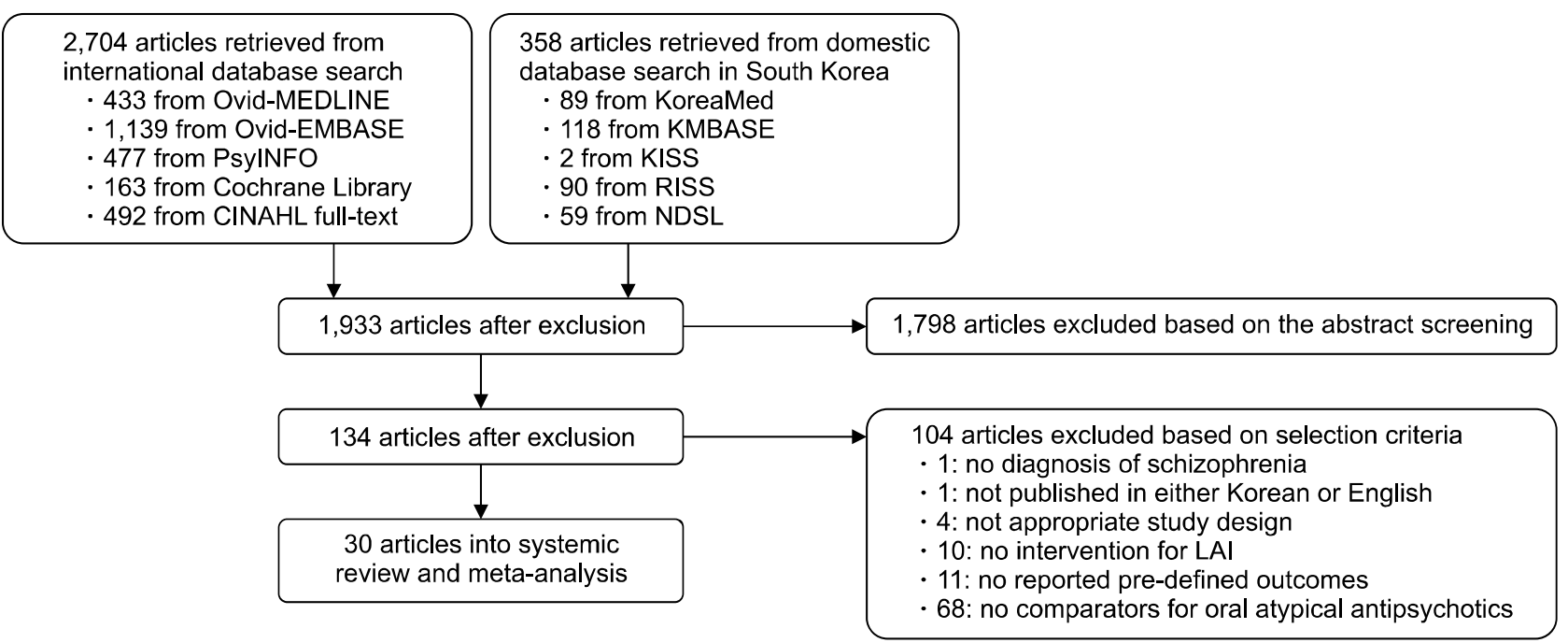

Fig. 1. Disposition of the selected articles for a systemic review and meta-analysis.

KMBASE, Korean Medicine Database; KISS, Korean Studies Information Service System; RISS, the Research Information Sharing Service; NDSL, National Digital Science Library (NDSL); LAI, long-acting injectable. 


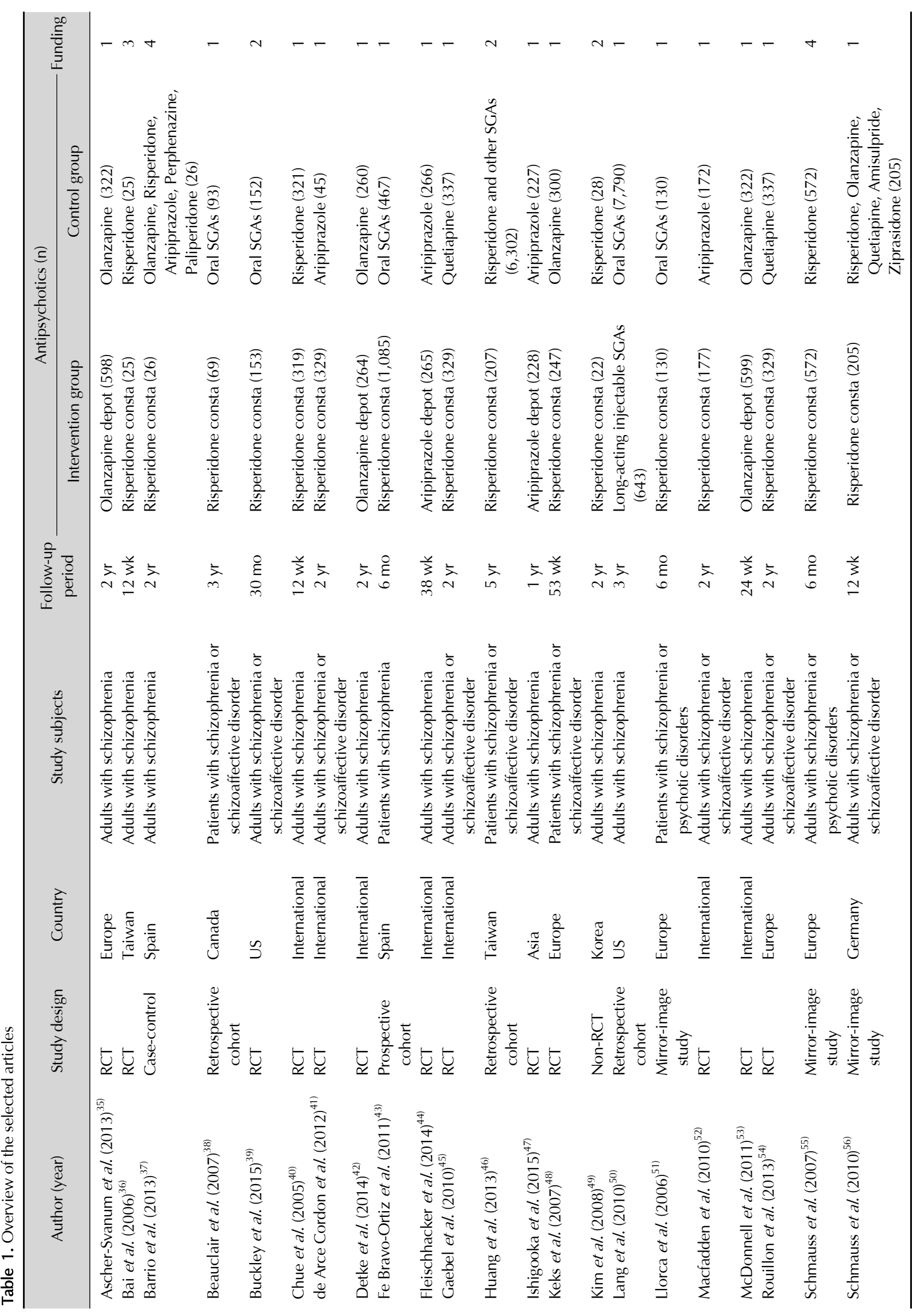




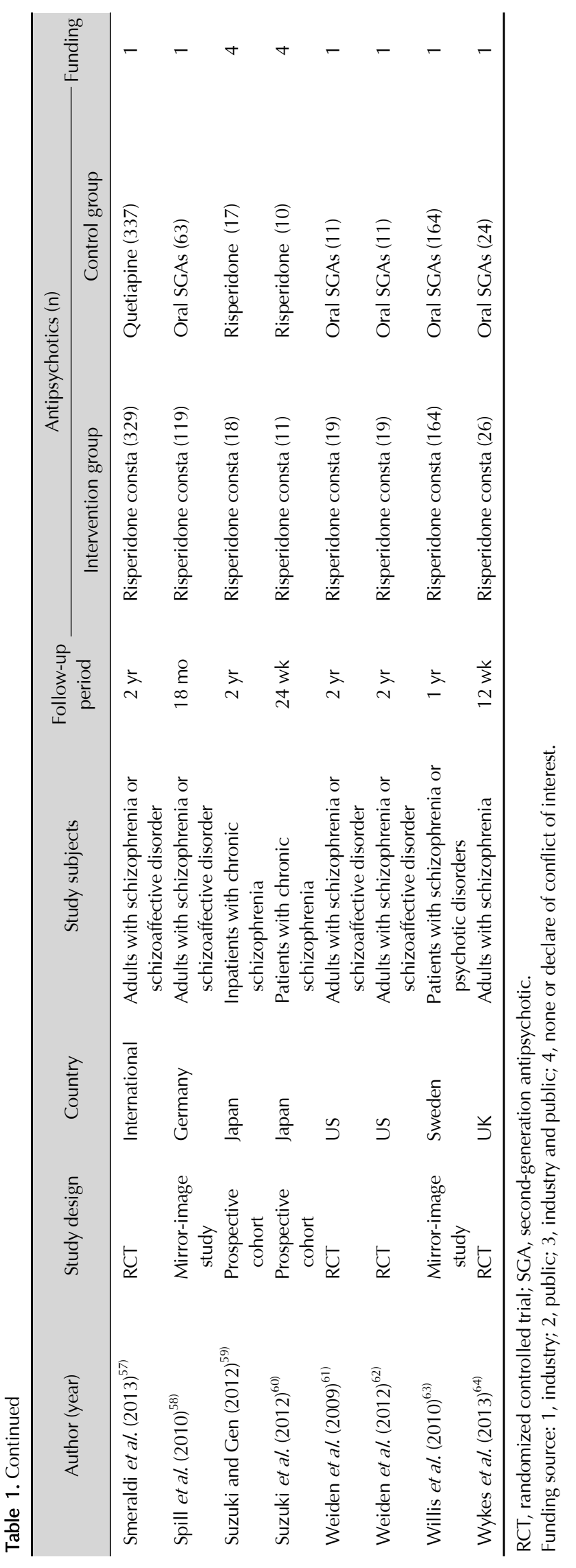


rate, remission rate, quality of life, hospital days, and other variables are shown in Table 2.

\section{Relapse Rate}

Because the findings of Rouillon et al. ${ }^{54)}$ and Gaebel et al. ${ }^{45)}$ were obtained from the same subjects, the relapse rate reported in Gaebel et al. ${ }^{45)}$ was considered a major measure and the findings in Rouillon et al. ${ }^{54)}$ were excluded from the meta-analysis. Ultimately, only six studies were used for this analysis. Overall, the group treated with LAI SGAs showed significantly lower relapse rates than did the oral SGAs group (RR, $0.85 ; 95 \% \mathrm{Cl}, 0.74$ to 0.99).

In the subgroup based on the length of the follow-up period, the relapse rate in the LAI SGAs group was $17 \%$ lower than that in the group treated with oral SGAs, in studies lasting $\geq 1$ year (RR, $0.83 ; 95 \% \mathrm{Cl}, 0.71$ to 0.97 ). However, there were no significant differences in relapse rates in studies lasting $\leq 1$ year $(\mathrm{RR}, 1.03 ; 95 \% \mathrm{Cl}, 0.66$ to 1.60). In addition, there were no significant differences between the groups with the relatively long and short follow-up periods $\left(\chi^{2}=0.78\right.$, degree of freedom $[d f]=1$, $p=0.38$ ) (Table 2).

\section{Non-compliance}

Although non-compliance was described in two articles, both of the articles investigated the same study subjects. Therefore, only the findings of Weiden et al. ${ }^{62)}$ were used and no meta-analyses were conducted. Weiden et $a l^{62)}$ compared non-compliance between schizophrenia patients treated with risperidone long-acting injection and oral SGAs. In their study, non-compliance was defined as "the complete discontinuation of all antipsychotic medication for more than 14 consecutive days.” There was no significant difference in non-compliance among patients treated for less than one year (RR, $0.58 ; 95 \% \mathrm{Cl}, 0.09$ to 3.35$)$, patients treated for one year (RR, $0.79 ; 95 \% \mathrm{Cl}, 0.47$ to 1.31 ), and patients treated for more than one year ( $R R, 0.92 ; 95 \% \mathrm{Cl}, 0.51$ to 1.66$)$ (Table 2).

\section{Time to Relapse}

Time to relapse (days) was reported in two articles. Both studies used risperidone long-acting injection as the intervention and conducted follow-up assessments for 24 months. In the study by de Arce Cordón et al., ${ }^{41)}$ the pro- portion of schizophrenia patients was $83 \%$ in the intervention group and $82.2 \%$ in the control group. In the study by Macfadden et al., ${ }^{52)}$ all the subjects were schizophrenia patients in the groups treated with $\mathrm{LAI}$ and oral SGAs. The mean disease duration of schizophrenia in the study by de Arce Cordón et al. ${ }^{41)}$ was 9.9 years in the group treated with LAI SGAs and 8.1 years in the group treated with oral SGA-treated group, respectively, the study of Macfadden et al., ${ }^{52)}$ included patients that experienced at least two episodes of relapse in the past two years as the study subjects. In results of the meta-analysis, we found that the group treated with LAI SGAs had significantly longer time to relapse than did the group treated with oral SGAs (SMD, 0.42; $95 \% \mathrm{Cl}, 0.29$ to 0.54) (Table 2).

\section{Hospitalization Rate}

Hospitalization rate was reported in three articles. Rehospitalization was defined as the subjects' psychiatric admission associated with psychotic and/or other psychiatric symptoms occurred after randomization in all studies. Weiden et al. ${ }^{62)}$ reported separate findings for one-year and two-year follow-up periods. In results of the metaanalysis, there was no statistically significant difference in the hospitalization rates of the groups treated with $\mathrm{LAI}$ and oral SGAs (RR, $0.83 ; 95 \% \mathrm{Cl}, 0.62$ to $1.11 ; I^{2}=29 \%$ ).

In the subgroup analysis, there were no significant differences according to the follow-up period. In addition, considering inter-group differences caused by the follow-up duration, there was no significant difference $\left(\chi^{2}=0.04, \mathrm{df}=1, p=0.84\right)$ (Table 2).

\section{Remission Rate}

Remission rate was reported in five articles. ${ }^{41,44,47,48,57)}$ Most of the studies used the schizophrenia remission criteria proposed by Andreasen et al., ${ }^{65)}$ although Keks et al. ${ }^{48)}$ defined remission as a $20 \%$ or more decline in the total PANSS score. Since the remission criteria of Andreasen et al. ${ }^{65)}$ and Keks et al. ${ }^{48)}$ were similar, in the data synthesis, the remission rates reported in each of the studies were used without modification. The meta-analysis showed no significant difference in remission rates for the groups treated with LAI or oral SGAs (RR, 1.07; 95\% $\mathrm{Cl}, 0.99$ to $1.15 ; \mathrm{I}^{2}=70.5 \%$ ).

In subgroup analysis, based on follow-up duration, the remission rate for the group treated with LAI SGAs was 


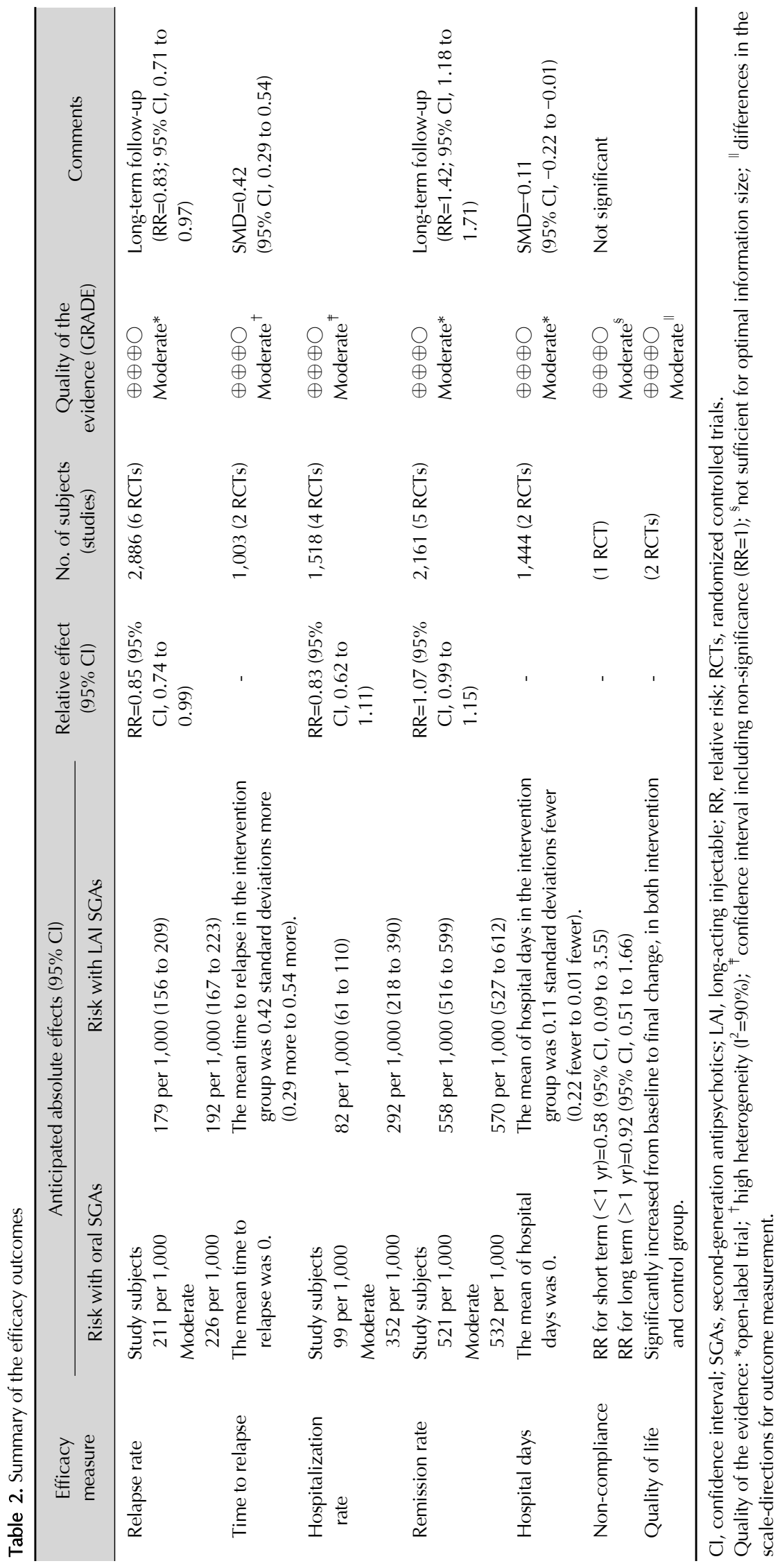


significantly greater than that for the oral SGAs group, in studies lasting $\geq 1$ year (RR, $1.42 ; 95 \% \mathrm{Cl}, 1.18$ to 1.71 ) (Table 2).

\section{Quality of Life}

Quality of life measures were reported in two articles. ${ }^{35,41)}$ Since the two studies used quite different assessment scales to measure quality of life, we could not synthesize the data quantitatively. In other words, the relatively high scores on the Schizophrenia Quality of Life Scale (SQLS) ${ }^{66)}$ which was used in the study by de Arce Cordón et al., ${ }^{41)}$ indicate a relatively low quality of life, whereas the relatively high scores in the Heinrichs Carpenter Quality of Life Scale (HQLS), ${ }^{67)}$ which was used in the study by Ascher-Svanum et al., ${ }^{35)}$ indicate a high quality of life. However, in all the groups treated with LAI and oral SGAs, the mean changes between the initial and final values were significant, and there were no inter-group comparisons (Table 2).

\section{Hospital Days}

"Hospital days" was defined as the duration of psychiatric admission associated with psychotic and/or other psychiatric symptoms in patients with schizophrenia. Two articles were included in the meta-analysis, and both studies used olanzapine long-acting injection as the intervention treatment. Detke et al. ${ }^{42)}$ evaluated hospital days associated with schizophrenia for a two-year follow-up period, and Ascher-Svanum et al. ${ }^{35)}$ evaluated hospital days for a six-month follow-up period. The mean hospital days for the group treated with LAI SGAs was significantly shorter than that for the oral SGAs group (SMD, -0.11 ; $95 \% \mathrm{Cl},-0.22$ to -0.01 ) (Table 2).

\section{Positive and Negative Syndrome Scale (PANSS)}

Total score on the PANSS was reported in nine studies included in the meta-analysis. The groups treated with LAI and oral SGAs were compared in terms of the mean change in the total score on the PANSS before and after SGA medications. In the meta-analysis, the decrease in total PANSS score in the group treated with LAI SGAs was greater than that in the oral SGAs group. However, this difference was not significant (SMD, $-0.05 ; 95 \% \mathrm{Cl},-0.12$ to 0.12$)$.

In subgroup analysis according to follow-up period, the decrease in total PANSS score was greater in the group treated with LAI SGAs than in the oral SGAs group. However, again, this difference was not significant. In addition, considering inter-group differences caused by the follow-up period, there was no significant difference $\left(\chi^{2}=1.59, \mathrm{df}=1, p=0.21\right)$ (Table 2).

\section{Clinical Global Impression Scale (CGI-S)}

Scores on the CGI-S were reported in eight studies included in the meta-analysis. The groups treated with LAI and oral SGAs were compared in terms of the mean change in score on the CGI-S before and after treatment with SGA medications. In the meta-analysis, the decrease in the CGI-S score in the group treated with LAI SGAs was greater than that in the oral SGAs group. However, this difference was not significant $(\mathrm{SMD},-0.05 ; 95 \% \mathrm{Cl},-0.13$ to $0.04 ; I^{2}=35.5 \%$ ).

In the subgroup analysis, according to follow-up period, there was no significant difference. In addition, considering inter-group differences caused by the follow-up period, there was no significant difference $\left(\chi^{2}=0.57, \mathrm{df}=1\right.$, $p=0.45$ ) (Table 2).

\section{Montgomery-Asberg Depression Rating Scale (MADRS)}

Scores on the MADRS were reported in two articles. Both studies were included in the ConstaTRE study. ${ }^{40)}$ de Arce Cordón et al. ${ }^{41)}$ used risperidone long-acting injection for intervention and oral aripiprazle for control and Smeraldi et al. ${ }^{57)}$ used risperidone long-acting injection for intervention and oral quetiapine for control for the follow-up period of 24 months. In the meta-analysis, the decrease in the MADRS score was significantly greater in the group treated with LAI SGAs than in the group treated with oral SGAs (SMD, $-1.69 ; 95 \% \mathrm{Cl},-2.95$ to -0.43 ) (Table 2).

\section{Safety Outcomes}

A summary of the safety outcomes, including all-cause discontinuation rate, drug-related side effects, ESRS, AIMS, and other side effects are shown in Table 3.

\section{All-cause Discontinuation Rate}

All-caused discontinuation rates were described in three articles included in the meta-analysis. Olanzapine long-acting injection was used in the study of Detke et al. ${ }^{42)}$ and aripiprazole long-acting injection was used in 


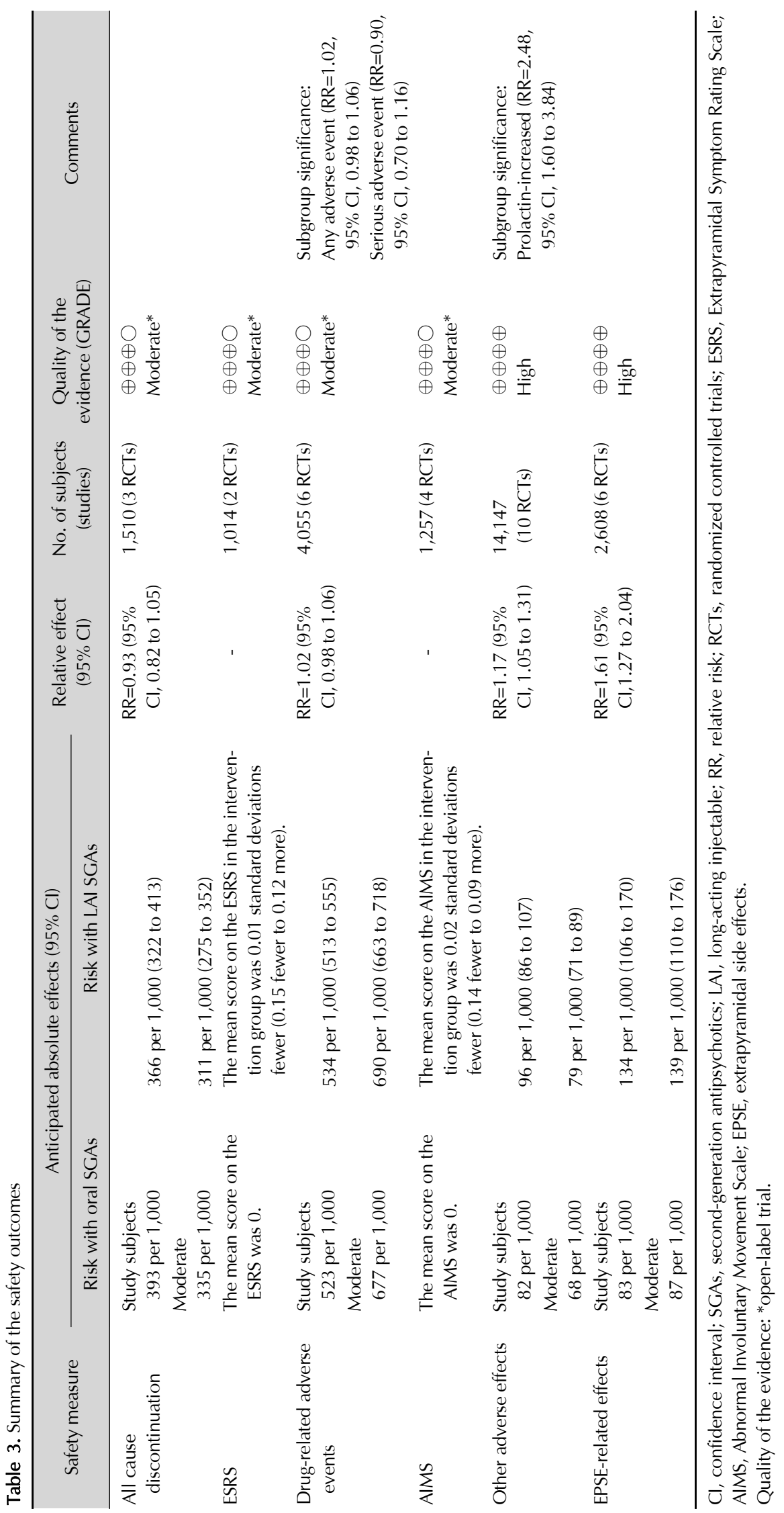


the other two studies. In the meta-analysis, the group treated with LAI SGAs had a lower discontinuation rate than did the oral SGAs group. However, this difference was not significant (RR, 0.93; 95\% Cl, 0.82 to 1.05 ) (Table 3).

\section{Extrapyramidal Symptom Rating Scale (ESRS)}

Scores on the ESRS were reported in two studies. In the meta-analysis, the decreased sore in the ESRS score was greater in the group treated with LAI SGAs than in the oral SGAs group (SMD, $-0.01 ; 95 \% \mathrm{Cl},-0.15$ to 0.12 ) (Table 3).

\section{Drug-related Side Effects}

In the meta-analysis, there was no statistically significant difference between the two groups in terms of all drug-related side effects ( $R R, 1.02 ; 95 \% \mathrm{Cl}, 0.98$ to 1.07 ) or severe drug-related side effects ( $R R, 0.90 ; 95 \% \mathrm{Cl}, 0.70$ to 1.16) (Table 3).

\section{Abnormal Involuntary Movement Scale (AIMS)}

Scores on the AIMS were reported in two studies. In the meta-analysis, the decrease in the AIMS score was greater in the group treated with LAI SGAs than in the oral SGAs group, although this difference was not statistically significant (SMD, $-0.02 ; 95 \% \mathrm{Cl},-0.14$ to 0.09 ) (Table 3 ).

\section{Other Adverse Effects}

Extrapyramidal side effects occurred significantly more often in the group treated with LAI SGAs than in the group treated with oral SGAs (RR, 1.61; 95\% Cl, 1.27 to 2.04), whereas there was no significant difference between the two groups in terms of all other drug-related adverse effects (Table 3). Prolactin-related side effects also occurred more often in the group treated with LAI SGAs than in the group treated with oral SGAs (RR, 2.48; 95\% Cl, 1.60 to 3.84). However, there was no significant difference between the two groups in terms of the incidence of other adverse effects, including akathisia, insomnia, and weight gain (Table 3).

\section{DISCUSSION}

In summary, with respect to outcome measures, the group treated with LAI SGAs was characterized by a significantly lower relapse rate, longer time to relapse, and fewer hospital days than the group treated with oral SGAs. However, there were no significant differences in hospitalization rate, remission rate, or non-compliance between the two groups. In terms of outcome measures, subgroup analysis limited to the studies lasting $\geq 1$ year showed that the group treated with LAI SGAs was characterized by significantly lower relapse rate and higher remission rate than the oral SGAs group. In addition, in terms of safety outcome measures, the group treated with LAI SGAs was characterized by higher rates of extrapyramidal syndrome and prolactin-related symptoms than the group treated with oral SGAs. However, there was no significant difference in the all-cause discontinuation rate between the two groups.

In the context of comparing the efficacy of LAI and oral antipsychotics in relapse prevention, the study findings were somewhat consistent as follows: Our study showed that the mean relapse prevention rate for schizophrenia patients treated with oral SGAs was significantly lower than that for patients treated with LAI SGAs. However, a meta-analysis limited to the studies lasting $\leq 1$ year showed that there were no significant differences in the relapse rate between patients treated with oral versus LAI SGAs. In findings similar to ours, superiority of LAI antipsychotics to oral antipsychotics for relapse prevention was supported by several naturalistic and mirror-image studies. ${ }^{68-73)}$ For example, the rehospitalization risk in schizophrenia or schizoaffective disorder patients treated with LAI antipsychotics was significantly lower than that in those patients treated with oral antipsychotics, in a nationwide cohort of the United States patients. ${ }^{70)}$ In addition, in a systematic review limited to mirror-image studies, ${ }^{71)}$ the group treated with LAI antipsychotics was characterized by significantly lower hospitalization rate than was the group treated with oral antipsychotics.

In contrast to our findings, a previous meta-analysis, which included 21 RCTs $(n=5,176)$ for both LAI FGAs and SGAs, ${ }^{11)}$ reported that there were no significant differences in the outcome measures, including relapse prevention, between the groups treated with $\mathrm{LAI}$ and oral antipsychotics (12 studies; RR, 0.93; 95\% Cl, 0.80 to 1.08; $p=0.35$ ). In the subgroup analyses confined to the FGAs, LAI formulations were characterized by greater relapse prevention than were oral formulations (10 studies; RR, $0.82 ; 95 \% \mathrm{Cl}, 0.65$ to $0.97 ; p=0.02$ ). Herein, Kishimoto et al. ${ }^{11)}$ explained that the findings could be associated with 
a publication bias and/or changes in relapse definitions, and the superiority of LAls to oral antipsychotics with respect to relapse prevention could not be clearly concluded. Moreover, whereas LAI antipsychotics are usually be prescribed to schizophrenia patients with non-compliance and greater illness severity in clinical psychiatry, LAI antipsychotics might also be prescribed to those with compliance and less illness severity. Herein, the RCTs for $\mathrm{LAI}$ and oral antipsychotics could present no significant differences in the efficacy in relapse prevention. ${ }^{72)}$ However, our findings showed that LAI SGAs had greater efficacy in terms of relapse prevention and were associated with higher rates of compliance in the relapse prevention than were oral SGAs, in patients with schizophrenia. The superior efficacy of LAI antipsychotics to oral antipsychotics was often shown in studies lasting more than 1 year, rather than in studies lasting less than 1 year. Leucht et al. ${ }^{8)}$ conducted a meta-analysis including only the LAI RCTs lasting more than 1 year and concluded that LAI antipsychotics result in greater relapse prevention than do oral antipsychotics in schizophrenia patients. In our systematic review and meta-analysis, most of the selected RCTs were continued for longer than 1 year. Moreover, differences in the study findings could be caused by variation in the definitions used to characterize relapse and hospitalization.

Kish et al. ${ }^{13)}$ found no significant differences in allcause death or suicide death between schizophrenia patients treated with $\mathrm{LAI}$ antipsychotics and placebo or oral antipsychotics in a meta-analysis including 52 RCTs. However, the authors speculated that extrapyramidal syndromes were more prevalent in the group treated with LAI antipsychotics, although the evidence was not well-captured by the meta-analysis. ${ }^{9)}$ There were no significant differences in extrapyramidal symptoms, prolactin increase, or other adverse events between the LAI and oral antipsychotics treatments in a meta-analysis of 18 RCTs, including data for risperidone, olanzapine, aripiprazole, zuclopenthixol, fluphenazine and haloperidol. ${ }^{17)}$ In contrast to these findings, a meta-analysis showed that LAI SGAs were characterized by a greater risk of developing extrapyramidal side effects than were LAI FGAs (RR, $1.451 ; p=0.048){ }^{23)}$ Moreover, our systematic review and meta-analysis have a virtue of presenting the evidence for significantly increased extrapyramidal syndrome and prolactin-related adverse effects in the group treated with LAI
SGAs compared with the group treated with oral SGAs. Herein, with the high level of evidence, our findings suggest that anticholinergic medications and other countermeasures against dopamine $D_{2}$ antagonism-related effects might be more necessary with LAI SGAs than with oral SGAs.

Several limitations of our study should be considered: First, the study subjects were limited to adults with schizophrenia. Second, in subgroup analysis, differences in the length of the follow-up period may contribute to high heterogeneity. The differences in disease severity in study subjects can be another contributor to high heterogeneity. Third, despite differences associated with the different pharmacological ingredients used in the intervention and control groups, we did not have a sufficient number of studies to enable a quantitative analysis of these differences. Fourth, cognitive symptoms were not included as a component of the efficacy outcomes, since the domain was not evaluated in previous clinical trials.

Despite these limitations, this systematic review and meta-analysis was able to demonstrate that LAI SGAs showed improved efficacy with respect to relapse rate, time to relapse, and hospital days, but also higher rates of dopamine $\mathrm{D}_{2}$ antagonism-related adverse effects, when compared with oral SGAs, with moderate to high levels of evidence. The superior efficacy of LAI SGAs to oral SGAs in our meta-analysis may be associated with the fact that most of the selected trials chosen for analysis continued for more than 1 year.

\section{- Acknowledgments}

This study was supported by a grant from the National Evidence-based Healthcare Collaborating Agency (NC15004).

\section{REFERENCES}

1. Collins PY, Patel V, Joestl SS, March D, Insel TR, Daar AS; Scientific Advisory Board and the Executive Committee of the Grand Challenges on Global Mental Health. Grand challenges in global mental health. Nature 2011;475:27-30.

2. Kim YK, Choi J, Park SC. A novel bio-psychosocial-behavioral treatment model in schizophrenia. Int J Mol Sci 2017;18: E734.

3. Karadag F, Sengul CB, Enli Y, Karakulah K, Alacam H, Kaptanoglu B, et al. Relationship between serum bilirubin levels and metabolic syndrome in patients with schizophrenia spectrum disorders. Clin Psychopharmacol Neurosci 2017; 
15:153-162.

4. Tripathi A, Kar SK, Shukla R. Cognitive deficits in schizophrenia: Understanding the biological correlates and remediation strategies. Clin Psychopharmacol Neurosci 2018; 16:7-17.

5. Corrigan PW, Liberman RP, Engel JD. From noncompliance to collaboration in the treatment of schizophrenia. Hosp Community Psychiatry 1990;41:1203-1211.

6. Novick D, Haro JM, Suarez D, Perez V, Dittmann RW, Haddad PM. Predictors and clinical consequences of non-adherence with antipsychotic medication in the outpatient treatment of schizophrenia. Psychiatry Res 2010;176:109-113.

7. Olfson M, Mechanic D, Hansell S, Boyer CA, Walkup J, Weiden PJ. Predicting medication noncompliance after hospital discharge among patients with schizophrenia. Psychiatr Serv 2000;51:216-222.

8. Leucht C, Heres S, Kane JM, Kissling W, Davis JM, Leucht S. Oral versus depot antipsychotic drugs for schizophrenia--a critical systematic review and meta-analysis of randomised long-term trials. Schizophr Res 2011;127:83-92.

9. Adams CE, Fenton MK, Quraishi S, David AS. Systematic meta-review of depot antipsychotic drugs for people with schizophrenia. Br J Psychiatry 2001;179:290-299.

10. Kishimoto T, Nitta M, Borenstein M, Kane JM, Correll CU. Long-acting injectable versus oral antipsychotics in schizophrenia: a systematic review and meta-analysis of mirror-image studies. J Clin Psychiatry 2013;74:957-965.

11. Kishimoto T, Robenzadeh A, Leucht C, Leucht S, Watanabe K, Mimura M, et al. Long-acting injectable vs oral antipsychotics for relapse prevention in schizophrenia: a meta-analysis of randomized trials. Schizophr Bull 2014;40:192-213.

12. Manchanda R, Chue P, Malla A, Tibbo P, Roy MA, Williams $\mathrm{R}$, et al. Long-acting injectable antipsychotics: evidence of effectiveness and use. Can J Psychiatry 2013;58(5 Supp/ 1): 5S-13S.

13. Kishi T, Matsunaga S, Iwata N. Mortality risk associated with long-acting injectable antipsychotics: a systematic review and meta-analyses of randomized controlled trials. Schizophr Bull 2016;42:1438-1445.

14. Kishi T, Oya K, Iwata N. Long-acting injectable antipsychotics for the prevention of relapse in patients with recent-onset psychotic disorders: a systematic review and meta-analysis of randomized controlled trials. Psychiatry Res 2016;246:750755.

15. Lafeuille MH, Dean J, Carter V, Duh MS, Fastenau J, Dirani R, et al. Systematic review of long-acting injectables versus oral atypical antipsychotics on hospitalization in schizophrenia. Curr Med Res Opin 2014;30:1643-1655.

16. Misawa F, Kishimoto T, Hagi K, Kane JM, Correll CU. Safety and tolerability of long-acting injectable versus oral antipsychotics: A meta-analysis of randomized controlled studies comparing the same antipsychotics. Schizophr Res 2016; 176:220-230.
17. Ostuzzi G, Bighelli I, So R, Furukawa TA, Barbui C. Does formulation matter? A systematic review and meta-analysis of oral versus long-acting antipsychotic studies. Schizophr Res 2017;183:10-21.

18. Meltzer HY, Matsubara S, Lee JC. Classification of typical and atypical antipsychotic drugs on the basis of dopamine D-1, D-2 and serotonin2 pKi values. J Pharmacol Exp Ther 1989; 251:238-246.

19. Fischer-Barnicol D, Lanquillon S, Haen E, Zofel P, Koch HJ, Dose M, et al; Working Group 'Drugs in Psychiatry'. Typical and atypical antipsychotics: the misleading dichotomy. Results from the Working Group 'Drugs in Psychiatry' (AGATE). Neuropsychobiology 2008;57:80-87.

20. Charych El, Liu F, Moss SJ, Brandon NJ. GABA(A) receptors and their associated proteins: implications in the etiology and treatment of schizophrenia and related disorders. Neuropharmacology 2009;57:481-495.

21. Gründer G, Hippius H, Carlsson A. The 'atypicality' of antipsychotics: a concept re-examined and re-defined. Nat Rev Drug Discov 2009;8:197-202.

22. Kim YS, Ahn YM, Jeong SH. Principle and practice of long-acting injectable antipsychotics. Seoul;Seoul National University Press;2010. p.40-41. Korean.

23. Fusar-Poli P, Kempton MJ, Rosenheck RA. Efficacy and safety of second-generation long-acting injections in schizophrenia: a meta-analysis of randomized-controlled trials. Int Clin Psychopharmacol 2013;28:57-66.

24. Huang X, Lin J, Demner-Fushman D. Evaluation of PICO as a knowledge representation for clinical questions. AMIA Annu Symp Proc 2006:359-363.

25. Tandon R, Nasrallah HA, Keshavan MS. Schizophrenia, “just the facts" 5. Treatment and prevention. Past, present, and future. Schizophr Res 2010;122:1-23.

26. Guy W. ECDEU assessment manual for psychopharmacology, Revised 1976. Rockville:National Institute for Mental Health; 1976 .

27. Kay SR, Fiszbein A, Opler LA. The Positive and Negative Syndrome Scale (PANSS) for schizophrenia. Schizophr Bull 1987; 13:261-276.

28. Montgomery SA, Asberg M. A new depression scale designed to be sensitive to change. Br I Psychiatry 1979;134:382-389.

29. Chouinard G, Margolese HC. Manual for the Extrapyramidal Symptom Rating Scale (ESRS). Schizophr Res 2005;76:247265.

30. Munetz MR, Benjamin S. How to examine patients using the Abnormal Involuntary Movement Scale. Hosp Community Psychiatry 1988;39:1172-1177.

31. Moher D, Shamseer L, Clarke M, Ghersi D, Liberati A, Petticrew M, et al; PRISMA-P Group. Preferred reporting items for systematic review and meta-analysis protocols (PRISMA-P) 2015 statement. Syst Rev 2015;4:1.

32. Higgins JPT, Green SS. Cochrane handbook for systematic reviews of interventions. Chichester:Wiley-Blackwell;2008. 
33. Kim SY, Park JE, Lee YJ, Seo HJ, Sheen SS, Hahn S, et al. Testing a tool for assessing the risk of bias for nonrandomized studies showed moderate reliability and promising validity. J Clin Epidemiol 2013;66:408-414.

34. Guyatt G, Oxman AD, Akl EA, Kunz R, Vist G, Brozek J, et al. GRADE guidelines: 1. Introduction-GRADE evidence profiles and summary of findings tables. J Clin Epidemiol 2011;64: 383-394.

35. Ascher-Svanum H, Novick D, Haro JM, Bertsch J, McDonnell $\mathrm{D}$, Detke H. Predictors of psychiatric hospitalization during 6 months of maintenance treatment with olanzapine long-acting injection: post hoc analysis of a randomized, double-blind study. BMC Psychiatry 2013;13:224.

36. Bai YM, Chen TT, Wu B, Hung CH, Lin WK, Hu TM, et al. $A$ comparative efficacy and safety study of long-acting risperidone injection and risperidone oral tablets among hospitalized patients: 12-week randomized, single-blind study. Pharmacopsychiatry 2006;39:135-141.

37. Barrio P, Batalla A, Castellví P, Hidalgo D, García M, Ortiz A, et al. Effectiveness of long-acting injectable risperidone versus oral antipsychotics in the treatment of recent-onset schizophrenia: a case-control study. Int Clin Psychopharmacol 2013;28:164-170.

38. Beauclair L, Chue P, McCormick J, Camacho F, Lam A, Luong D. Impact of risperidone long-acting injectable on hospitalisation and medication use in Canadian patients with schizophrenia. J Med Econ 2007;10:427-442.

39. Buckley PF, Schooler NR, Goff DC, Hsiao J, Kopelowicz A, Lauriello J, et al; PROACTIVE Study. Comparison of SGA oral medications and long-acting injectable SGA: the PROACTIVE study. Schizophr Bull 2015;41:449-459.

40. Chue P, Eerdekens M, Augustyns I, Lachaux B, Molcan P, Eriksson L, et al. Comparative efficacy and safety of long-acting risperidone and risperidone oral tablets. Eur Neuropsychopharmacol 2005; 15:111-117.

41. de Arce Cordón R, Eding E, Marques-Teixeira J, Milanova V, Rancans E, Schreiner A. Descriptive analyses of the aripiprazole arm in the risperidone long-acting injectable versus quetiapine relapse prevention trial (ConstaTRE). Eur Arch Psychiatry Clin Neurosci 2012;262:139-149.

42. Detke HC, Weiden PJ, Llorca PM, Choukour M, Watson SB, Brunner $\mathrm{E}$, et al. Comparison of olanzapine long-acting injection and oral olanzapine: a 2-year, randomized, open-label study in outpatients with schizophrenia. I Clin Psychopharmacol 2014;34:426-434.

43. Fe Bravo-Ortiz M, Gutiérrez-Casares JR, Rodríguez-Morales A, García MA, Hidalgo-Borrajo R. Influence of type of treatment on the well-being of Spanish patients with schizophrenia and their caregivers. Int I Psychiatry Clin Pract 2011; 15:286-295.

44. Fleischhacker WW, Sanchez R, Perry PP, Jin N, PetersStrickland T, Johnson BR, et al. Aripiprazole once-monthly for treatment of schizophrenia: double-blind, randomised, non- inferiority study. Br J Psychiatry 2014;205:135-144.

45. Gaebel W, Schreiner A, Bergmans P, de Arce R, Rouillon F, Cordes J, et al. Relapse prevention in schizophrenia and schizoaffective disorder with risperidone long-acting injectable vs quetiapine: results of a long-term, open-label, randomized clinical trial. Neuropsychopharmacology 2010;35: 2367-2377.

46. Huang SS, Lin $\mathrm{CH}$, Loh el-W, Yang HY, Chan $\mathrm{CH}$, Lan TH. Antipsychotic formulation and one-year rehospitalization of schizophrenia patients: a population-based cohort study. Psychiatr Serv 2013;64:1259-1262.

47. Ishigooka J, Nakamura J, Fujii Y, Iwata N, Kishimoto T, Iyo M, et al; ALPHA Study Group. Efficacy and safety of aripiprazole once-monthly in Asian patients with schizophrenia: a multicenter, randomized, double-blind, non-inferiority study versus oral aripiprazole. Schizophr Res 2015;161:421-428.

48. Keks NA, Ingham M, Khan A, Karcher K. Long-acting injectable risperidone $v$. olanzapine tablets for schizophrenia or schizoaffective disorder. Randomised, controlled, open-label study. Br J Psychiatry 2007; 191:131-139.

49. Kim B, Lee SH, Choi TK, Suh S, Kim YW, Lee E, et al. Effectiveness of risperidone long-acting injection in first-episode schizophrenia: in naturalistic setting. Prog Neuropsychopharmacol Biol Psychiatry 2008;32:1231-1235.

50. Lang K, Meyers JL, Korn JR, Lee S, Sikirica M, Crivera C, et al. Medication adherence and hospitalization among patients with schizophrenia treated with antipsychotics. Psychiatr Serv 2010;61:1239-1247.

51. Llorca PM, Kahn JP, Moreau-Mallet V, Bouhours P, The French StoRMi Investigators Group. Symptom control, functioning, and hospitalization status in French patients changed from oral atypical antipsychotics to risperidone long-acting injectable. Int I Psychiatry Clin Pract 2006;10:276-284.

52. Macfadden W, Ma YW, Thomas Haskins J, Bossie CA, Alphs L. A prospective study comparing the long-term effectiveness of injectable risperidone long-acting therapy and oral aripiprazole in patients with schizophrenia. Psychiatry (Edgmont) 2010;7:23-31.

53. McDonnell DP, Kryzhanovskaya LA, Zhao F, Detke HC, Feldman PD. Comparison of metabolic changes in patients with schizophrenia during randomized treatment with intramuscular olanzapine long-acting injection versus oral olanzapine. Hum Psychopharmacol 2011;26:422-433.

54. Rouillon F, Eriksson L, Burba B, Raboch J, Kaprinis G, Schreiner A. Functional recovery results from the risperidone long-acting injectable versus quetiapine relapse prevention trial (ConstaTRE). Acta Neuropsychiatr 2013;25:297-306.

55. Schmauss M, Sacchetti E, Kahn JP, Medori R. Efficacy and safety of risperidone long-acting injectable in stable psychotic patients previously treated with oral risperidone. Int Clin Psychopharmacol 2007;22:85-92.

56. Schmauss M, Diekamp B, Gerwe M, Schreiner A, Ibach B. Does oral antipsychotic pre-treatment influence outcome of a 
switch to long-acting injectable risperidone in patients with schizophrenia? Pharmacopsychiatry 2010;43:73-80.

57. Smeraldi E, Cavallaro R, Folnegović-Šmalc V, Bidzan L, Emin Ceylan M, Schreiner A. Long-term remission in schizophrenia and schizoaffective disorder: results from the risperidone long-acting injectable versus quetiapine relapse prevention trial (ConstaTRE). Ther Adv Psychopharmacol 2013;3:191199.

58. Spill B, Konoppa S, Kissling W, Maino K, Messer T, Heres S. Long-term observation of patients successfully switched to risperidone long-acting injectable: A retrospective, naturalistic 18-month mirror-image study of hospitalization rates and therapy costs. Int I Psychiatry Clin Pract 2010;14:53-62.

59. Suzuki $\mathrm{H}$, Gen K. The influence of switching from oral risperidone to risperidone long-acting injection on the clinical symptoms and cognitive function in schizophrenia. Ther $A d v$ Psychopharmacol 2012;2:23-29.

60. Suzuki H, Inoue Y, Gen K. A study of the efficacy and safety of switching from oral risperidone to risperidone long-acting injection in older patients with schizophrenia. Ther $\mathrm{Ad} v$ Psychopharmacol 2012;2:227-234.

61. Weiden PJ, Schooler NR, Weedon JC, Elmouchtari A, Sunakawa A, Goldfinger SM. A randomized controlled trial of long-acting injectable risperidone vs continuation on oral atypical antipsychotics for first-episode schizophrenia patients: initial adherence outcome. J Clin Psychiatry 2009;70: 1397-1406.

62. Weiden PJ, Schooler NR, Weedon JC, Elmouchtari A, Sunakawa-McMillan A. Maintenance treatment with long-acting injectable risperidone in first-episode schizophrenia: a randomized effectiveness study. J Clin Psychiatry 2012; 73:1224-1233.

63. Willis $M$, Svensson $M$, Löthgren $M$, Eriksson B, Berntsson A, Persson U. The impact on schizophrenia-related hospital utilization and costs of switching to long-acting risperidone injections in Sweden. Eur J Health Econ 2010;11:585-594.

64. Wykes T, Rose D, Williams P, David AS. Working alliance and its relationship to outcomes in a randomized controlled trial (RCT) of antipsychotic medication. BMC Psychiatry 2013;13:28.

65. Andreasen NC, Carpenter WT Jr, Kane JM, Lasser RA, Marder SR, Weinberger DR. Remission in schizophrenia: proposed criteria and rationale for consensus. Am I Psychiatry 2005; 162:441-449.

66. Martin CR, Allan R. Factor structure of the Schizophrenia Quality of Life Scale Revision 4 (SQLS-R4). Psychol Health Med 2007; 12:126-134.

67. Heinrichs DW, Hanlon TE, Carpenter WT Jr. The Quality of Life Scale: an instrument for rating the schizophrenic deficit syndrome. Schizophr Bull 1984;10:388-398.

68. Lambert T, Olivares JM, Peuskens J, DeSouza C, Kozma CM, Otten $\mathrm{P}$, et al. Effectiveness of injectable risperidone long-acting therapy for schizophrenia: data from the US, Spain, Australia, and Belgium. Ann Gen Psychiatry 2011;10:10.

69. Peng X, Ascher-Svanum H, Faries D, Conley RR, Schuh KJ. Decline in hospitalization risk and health care cost after initiation of depot antipsychotics in the treatment of schizophrenia. Clinicoecon Outcomes Res 2011;3:9-14.

70. Tiihonen J, Wahlbeck K, Lönnqvist J, Klaukka T, loannidis JP, Volavka J, et al. Effectiveness of antipsychotic treatments in a nationwide cohort of patients in community care after first hospitalisation due to schizophrenia and schizoaffective disorder: observational follow-up study. BMJ 2006;333:224.

71. Tiihonen J, Haukka J, Taylor M, Haddad PM, Patel MX, Korhonen P. A nationwide cohort study of oral and depot antipsychotics after first hospitalization for schizophrenia. Am J Psychiatry 2011;168:603-609.

72. Grimaldi-Bensouda L, Rouillon F, Astruc B, Rossignol M, Benichou J, Falissard B, et al; CGS Study Group. Does longacting injectable risperidone make a difference to the real-life treatment of schizophrenia? Results of the Cohort for the General study of Schizophrenia (CGS). Schizophr Res 2012; 134:187-194.

73. Correll CU, Kishimoto T, Kane JM. Randomized controlled trials in schizophrenia: opportunities, limitations, and trial design alternatives. Dialogues Clin Neurosci 2011;13:155-172. 